\title{
Patterned activity within the local cortical architecture
}

\author{
Farran Briggs and W. Martin Usrey* \\ Center for Neuroscience, University of California, Davis, CA, USA
}

The cerebral cortex is a vastly complex structure consisting of multiple distinct populations of neurons residing in functionally specialized cortical compartments. A fundamental goal in systems neuroscience is to understand the interactions among cortical neurons and their relationship to behavior. It is hypothesized that dynamic activity patterns, such as oscillations in global neuronal activity, could span large, heterogeneous populations of cortical neurons in such a manner as to bind together the activity of otherwise disparate cortical networks. Little is known about the mechanisms by which such global oscillatory patterns entrain cortical networks, or the contribution of such activity patterns to cortical function. An important step toward elucidating the role of such patterned activity in cortical information processing is understanding these interactions at the local circuit level. Here, we highlight recent findings that provide insight into how dynamic activity patterns affect specific neuronal populations and circuits.

Keywords: gamma-band, oscillation, cortical circuit, geniculocortical, corticogeniculate

Edited by:

Rui M. Costa, Instituto Gulbenkian de Ciência, Portugal

Reviewed by:

Jose-Manuel Alonso, State University of New York, USA

${ }^{*}$ Correspondence:

W. Martin Usrey is a Professor of Neurobiology, Physiology and Behavior and Neurology at the University of California, Davis. He trained at Duke University, Rockefeller University and Harvard Medical School. His research focuses on understanding neuronal circuits for vision, with an emphasis on determining the functional relationship between feedforward and feedback pathways interconnecting the visual cortex and thalamus.

wmusrey@ucdavis.edu

\section{INTRODUCTION}

A hallmark of the mammalian brain is specificity. Distinct neuronal populations participate in specific circuits carrying different pieces of information in separated channels. These channels converge in specialized cortical areas, each containing a distinct architecture of local cortical circuitry where information is combined and sorted again into new channels relayed to the next cortical area, and so on. There is ample crosscommunication between distinct brain regions providing an anatomical substrate for sharing information throughout the brain. However, our cognitive experience is not a combination of pieces. Rather we perceive and act in the world in a continuously coordinated and coherent fashion. A fundamental question in neuroscience is: how can we explain the apparent paradox between a brain seemingly built to read-in and process bits of information, and an experience that is coherent.

One of the more robust phenomena in the mammalian brain is the coordinated activity of neurons. During such periods of activity, large heterogeneous populations of neurons spanning hundreds of microns of cortical space and a variety of brain areas modulate their electrical activity in rhythm with one another (see for review Singer, 1993; Steriade, 2001; Uhlhaas et al., 2009). This patterned or synchronized activity can occur on different time scales from slow/low periodicity $(<1 \mathrm{~Hz})$ to very fast/high periodicity $(200 \mathrm{~Hz})$. Periodic activity within the gamma frequency band (or gamma-band activity, spanning roughly 20 to $100 \mathrm{~Hz}$ ) has been implicated in processing sensory information as well as mediating cognitive influences such as attentional control (see for review Womelsdorf and Fries, 2007; Berens et al., 2008; Fries, 2009). Often, neurons participating in synchronized oscillations will respond within the same phase of the oscillation, suggesting the presence of a generator signal that entrains the population into the specific rhythm (see for review Fries, 2009; Haider and McCormick, 2009; Uhlhaas et al., 2009). Because these coordinated activity patterns have the potential to bind together 
Local cortical circuitry

Networks of interconnected neurons within a cortical area, often spanning many cortical layers and columnar compartments. Local cortical networks consist of multiple distinct neuronal cell types, including excitatory and inhibitory neurons.

Gamma-band activity

Oscillations in neuronal activity with a periodicity of about 20 to $100 \mathrm{~Hz}$. Measurements of gamma-band activity are usually taken from local-field potential recordings representing large populations (spanning 200-1000 microns), but can also be measured from spiking activity, membrane potential fluctuations, or intracellularly recorded currents.

\section{Geniculocortical}

Afferent thalamocortical connection from the lateral geniculate nucleus (LGN) of the thalamus to its cortical target, primary visual cortex (V1). Geniculocortical afferents are organized into parallel pathways that provide the cortex with specific types of visual information. Synapses from geniculocortical axons are made in specific sublaminar compartments within layers 4 and 6 of V1.

\section{Corticogeniculate}

Efferent corticothalamic connection from primary visual cortex (V1) to the lateral geniculate nucleus (LGN) of the thalamus. Corticogeniculate neurons reside in layer 6 of $\mathrm{V} 1$. In primates, the corticogeniculate pathway contains distinct populations of neurons with physiological properties that resemble those of neurons in the geniculocortical parallel pathways. different populations of neurons, even those separated by large distances, hypotheses have emerged suggesting that coordinated activity is one of the means by which the brain combines information into coherent percepts (Singer, 1993; Fries, 2009). Before such ideas are embraced, the mechanisms of coordinated activity and their functional contribution to cognition must be elucidated. In the sections below we describe recent findings aimed at elucidating the mechanisms and functional implications of coordinated activity patterns in specific cortical neurons and local circuits. We focus on neurons in the early visual system, since much is known about the anatomy, physiology and local circuitry of this population. Taken together, recent results from our laboratory and others suggest that coordinated activity patterns are likely to impact and interact differently with different types of neuronal networks. Thus, just as the cortex is characterized by specificity and complexity, it is likely that coordinated activity patterns play a variety of roles in cortical information processing depending on the circuits with which they interact.

\section{CIRCUITRY OF THE EARLY VISUAL SYSTEM}

To examine the effects of coordinated activity patterns on specific, identified cortical circuits, a model system is required where the anatomy and physiology of neurons participating in these circuits are well understood. The early visual system of nonhuman primates provides such a model, since much is known about the cellular anatomy and physiology of neurons connecting the retina, lateral geniculate nucleus of the thalamus (LGN), and primary visual cortex (V1; see for review, Callaway, 2004, 2005; Sincich and Horton, 2005). Using the macaque monkey, we focused on two specific populations of V1 neurons with distinct input/output circuit relations with the LGN: layer 4 geniculocortical input neurons and layer 6 corticogeniculate output, or feedback neurons (Figure 1).

In the early visual system, LGN neurons are the major source of visual information sent to V1 (see for review, Callaway, 2005). Most geniculate inputs terminate in layer 4 of V1 with some collateral input impinging upon layer 6, the deepest cortical layer (Hendrickson et al., 1978; Blasdel and Lund, 1983). LGN neurons carry relatively low-level visual information, responding preferentially to small spots of light, similar to the responses of retinal cells (see for review, Reid and Usrey, 2004). Once these signals arrive in V1, they are combined to increase the selectivity of cortical responses to specific features of the visual stimulus, including selectivity for stimulus orientation and direction of movement (Reid and Alonso, 1995; Reid and Usrey, 2004).
The local circuit architecture of V1 is vastly complex such that visual information is combined to varying extents before being passed on to higher visual cortical areas (Callaway, 2004; Sincich and Horton, 2005). However, some local cortical circuits are better understood, including those interconnecting layer 4 (the major target of LGN input) with layer 6 (the only layer providing corticogeniculate output) (see for review Briggs and Usrey, 2008; Briggs, 2010). Because layer 6 neurons project to both the LGN and the overlying cortical layer 4, they are in a unique position to influence feedforward input to V1 at two levels. In addition, a subset of corticogeniculate neurons in layer 6 receive direct, monosynaptic input from the LGN, providing a disynaptic loop by which visual information can rapidly travel between the LGN and V1 (Briggs and Usrey, 2007a). Although the details of how corticogeniculate neurons influence visual processing in the LGN have yet to be determined, it is clear that corticogeniculate circuits are organized into parallel channels that preserve the physiological features of the geniculocortical channels (Fitzpatrick et al. 1994; Briggs and Usrey, 2009a).

\section{PERIODIC ACTIVITY IN GENICULOCORTICAL, CORTICOGENICULATE, AND LOCAL CORTICAL CIRCUITS}

We examined the patterns of periodic activity in geniculocortical and corticogeniculate circuits to determine how these activity patterns affect neuronal communication within the early visual system. Within this framework, we studied layer 4 input neurons to understand how ongoing activity patterns affect geniculocortical communication. We also examined activity patterns of layer 6 corticogeniculate neurons to gain insight into the type of signals relayed from V1 to the LGN. Finally, we compared the spectral properties of periodic activity among layer 4 input neurons and layer 6 output neurons to determine the contributions made by the local cortical architecture toward patterned activity.

To understand how patterned activity modulates the activity of individual, identified neurons participating in known cortical circuits, we measured the spiking responses of identified layer 4 input neurons and layer 6 feedback neurons (Briggs and Usrey, 2007b, 2009b). Neurons were recorded in vivo in anesthetized and awakebehaving animals using extracellular electrodes, and identified physiologically by verifying their connectivity via orthodromic or antidromic stimulation within the LGN. For each recorded neuron, patterned activity was assessed by measuring the power spectra of spiking autocorrelograms. In addition to measuring patterns in 


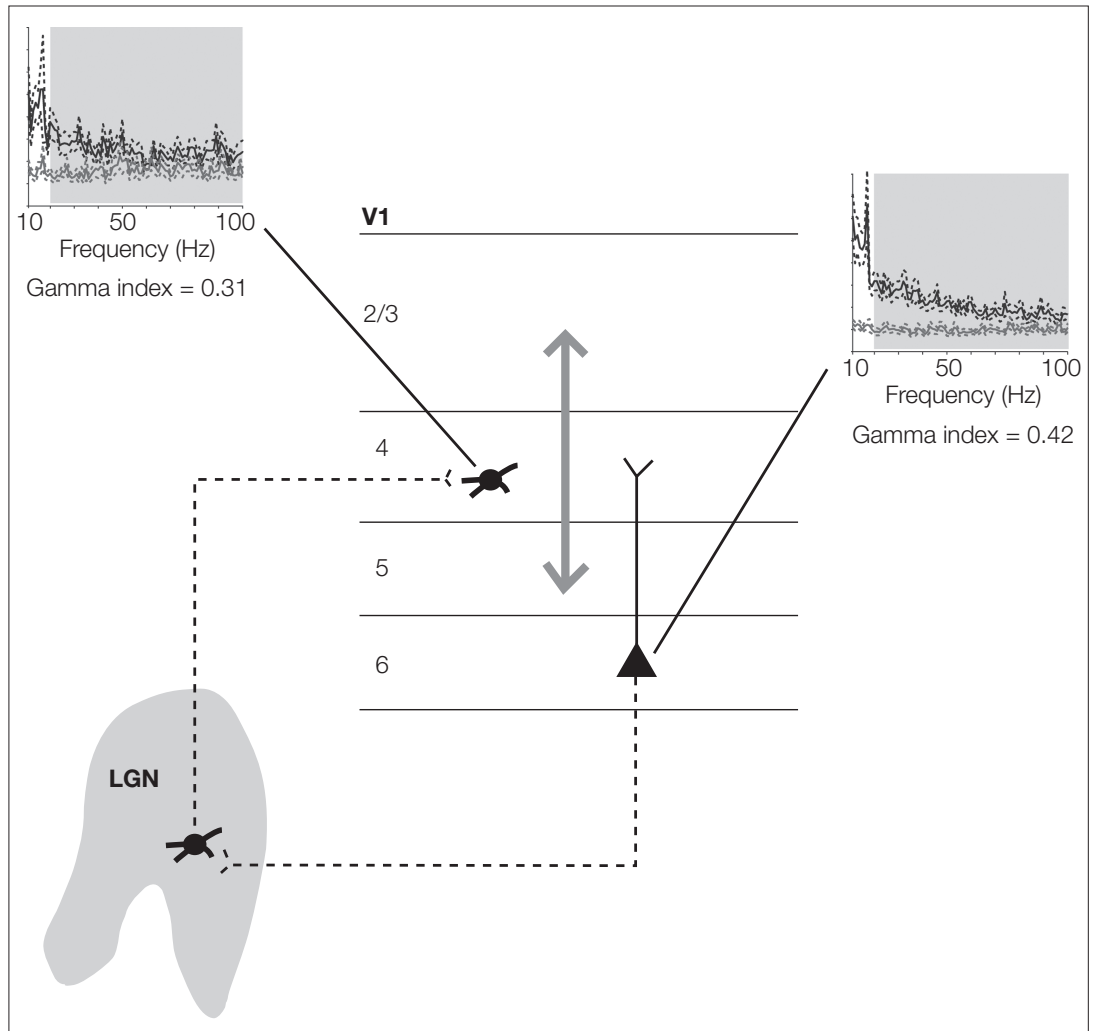

FIGURE 1 | Simplified schematic of geniculocortical, corticogeniculate, and local cortical circuits interconnecting neurons in the LGN and V1. Dendrites are depicted by solid lines and axons are depicted by dashed lines. The gray arrow represents a host of local cortical circuits within V1. Power spectra illustrate average power in autocorrelograms (black lines) and shuffled correlograms (gray lines) for layer 4 input neurons and layer 6 feedback neurons (dashed lines represent standard error). The extent to which the black lines lie above the gray lines indicates the amount of spectral power beyond that expected from firing rate alone (quantified with the gamma index). Light gray boxes outline the gamma-frequency band. Numbers beneath the power spectra indicate average gamma index values across samples of layer 4 input neurons and layer 6 output neurons (adapted from Briggs and Usrey, 2009a). that ongoing activity oscillating around $40 \mathrm{~Hz}$, i.e., within the gamma-band, had a deterministic effect on the efficacy of geniculocortical communication. Furthermore, when we examined the timing or phase of the oscillatory activity relative to the timing of the stimulated input, we found that electrically-evoked inputs were most effective when they occurred in phase with the gamma oscillation. These results suggest that a possible mechanism by which gamma oscillations may modulate neuronal activity is that they adjust the efficacy of synaptic inputs.

In a second set of experiments, we addressed the question of whether or not neurons at different levels within the local cortical architecture in V1 share similar or distinct patterns of periodic activity. In particular, we examined the relative amounts of gamma-band activity present in the spiking activity of layer 4 input neurons and layer 6 feedback neurons in V1 of awake-behaving monkeys (Figure 1; Briggs and Usrey, 2009b). We discovered that both layer 4 input neurons and layer 6 feedback neurons within V1 displayed significant beta- and gammaband oscillations in their ongoing spiking activity. Interestingly, layer 6 neurons consistently demonstrated increased gamma-band activity, especially at frequencies above $30 \mathrm{~Hz}$. Importantly, the increase in gamma-band activity in layer 6 neurons was not due to a change in firing rate, as both layer 4 and layer 6 neurons had statistically similar firing rates. The results from this set of experiments demonstrate that gamma-band oscillations differentially modulate distinct populations of cortical neurons. Furthermore, these results are consistent with a role for local cortical circuits in mediating the patterns of ongoing activity present within neuronal populations.

Much previous work has examined the interactions between thalamic and cortical structures in communicating and maintaining oscillations in ongoing activity. Some results suggest that the cortex controls rhythm generation in thalamic networks (Destexhe et al., 1999; Bal et al., 2000; Blumenfeld and McCormick, 2000; Destexhe, 2000; Steriade, 2001), and others have proposed that the thalamus could mediate zero phase-lagged synchronization of rhythms across cortical areas (Vicente et al., 2008; Gollo et al., 2010). Our results support the notion that the cortex conveys oscillatory structure to thalamic neurons; however, more work is required to determine whether this structure serves as the generator of a rhythmic signal.

\section{PATTERNED ACTIVITY AND CORTICAL NETWORK OPERATION}

Much recent work - including our own suggests that patterns of rhythmic activity differentially affect different populations of neurons. 
Conversely, increasing studies suggest that distinct neuronal populations and organizational structures are necessary to produce patterned activity in the cortex. All of these findings point toward the notion that coordinated patterns of activity are likely specific phenomena with distinct applications toward cognitive processing.

In corroboration with our results, suggesting that distinct neuronal populations participate in different types of coordinated activity patterns, a number of other studies have identified differences in oscillatory patterns across the cortical lamina (Kramer et al., 2008; Roopun et al., 2008; Sun and Dan, 2009). For instance, results from Sun and Dan demonstrate that oscillations in spontaneous synaptic currents have increased power at high frequencies in deep layer cortical neurons compared to neurons in more superficial cortical layers (Sun and Dan, 2009). Along these lines, Otte et al. (2010) provide evidence that different neuronal cell types exhibit different responses to simulated oscillatory input in the gamma-band, and that the response variations can be accounted for by the different kinetics of each neuronal type. There is also increasing evidence suggesting that fast-spiking, parvalbumin- positive inhibitory neurons in cortex are required for gamma-band oscillations, and that this population of inhibitory interneurons is uniquely well-equipped for the job given their characteristic short decay time constant and after-hyperpolarization latency (Bartos et al., 2007; Cardin et al., 2009; Sohal et al., 2009; Otte et al., 2010). Finally, while global oscillations may spread across wide regions of the cortex, increasing evidence suggests that specific cortical architectures may be required for specific oscillatory patterns, such as zero phase-lagged synchronization of gamma-band activity (Bazhenov et al., 2008; Yang et al., 2009).

In closing, while the larger question of what role coordinated activity patterns play in cognitive processes remains, increasing evidence suggests that ongoing activity is unlikely to play one singular role in information processing, but rather a variety of roles specialized for the neuronal populations and cortical circuits involved.

\section{ACKNOWLEDGMENTS}

This work was supported by NIH grants EY018683 (Farran Briggs) and EY 013588 (W. Martin Usrey).

\section{REFERENCES}

Bal, T., Debay, D., and Destexhe, A. (2000). Cortical feedback controls the frequency and synchrony of oscillations in the visual thalamus. J. Neurosci. 20, 7478-7488.

Bartos, M., Vida, I., and Jonas, P. (2007). Synaptic mechanisms of synchronized gamma oscillations in inhibitory interneuron networks. Nat. Rev. Neurosci. 8, 45-56.

Bazhenov, M., Rulkov, N. F., and Timofeev, I. (2008). Effect of synaptic connectivity on long-range synchronization of fast cortical oscillations. J. Neurophys. 100, 1562-1575.

Berens, P, Keliris, G. A., Ecker, A. S., Logothetis, N. K., and Tolias, A. S. (2008). Feature selectivity of the gamma-band of the local field potential in primate primary visual cortex. Front. Neurosci. 2:37 doi:10.3389/neuro.01.037.2008.

Blasdel, G. G., and Lund, J. S. (1983). Termination of afferent axons in macaque striate cortex. J. Neurosci. 3 , 1389-1413.

Blumenfeld, H., and McCormick, D. A. (2000). Corticothalamic inputs control the pattern of activity generated in thalamocortical networks. J. Neurosci. 20, 5153-5162.

Briggs, F. (2010). Organizing principles of cortical layer 6. Front. Neural. Circuits. doi:10.3389/neuro.04.003.2010.
Briggs, F., and Usrey, W. M. (2007a). A fast, reciprocal pathway between the lateral geniculate nucleus and visual cortex in the macaque monkey. J. Neurosci. 27, 5431-5436.

Briggs, F., and Usrey, W. M. (2007b). Cortical activity influences geniculocortical spike efficacy in the macaque monkey. Front. Integr. Neurosci. 1:3, doi: 10.3389/neuro.07/003.2007.

Briggs, F., and Usrey, W. M. (2008). Emerging views of corticothalamic function. Curr. Opin. Neurobiol. 18, 403-407.

Briggs, F., and Usrey, W. M. (2009a). Parallel processing in the corticogeniculate pathway of the macaque monkey. Neuron 62, 135-146.

Briggs, F., and Usrey, W. M. (2009b). Modulation of gamma-band activity across local cortical circuits. Front. Integr. Neurosci. 3:15. doi: 10.3389/neuro.07.015.2009.

Callaway, E. M. (2004). "Cell types and local circuits in primary visual cortex of the macaque monkey," in The Visual Neurosciences, Vol. 1, eds L. Chalupa and J. Werner (Cambridge, MA: MIT Press), 680-694.

Callaway, E. M. (2005). Structure and function of parallel pathways in the primate early visual system. J. Physiol. 566, 13-19.

Cardin, J. A., Carlen, M., Meletis, K., Knoblick, U., Zhang, F., Deisseroth,
K., Tsai, L-H., and Moore, C. I. (2009). Driving fast-spiking cells induces gamma rhythm and controls sensory responses. Nature 459 663-667.

Destexhe, A. (2000). Modelling corticothalamic feedback and the gating of the thalamus by cerebral cortex. $J$. Physiol. (Paris) 94, 391-410.

Destexhe, A., Contreras, D., and Steriade, M. (1999). Cortically-induced coherence of a thalamic-generated oscillation. J. Neurosci. 92, 427-443.

Fitzpatrick, D., Usrey, W. M., Schofield, B. R., and Einstein, G. (1994). The sublaminar organization of neurons in layer 6 of macaque striate cortex. Vis. Neurosci. 11, 307-315.

Fries, P. (2009). Neuronal gamma-band synchronization as a fundamental process in cortical computation. Annu. Rev. Neurosci. 32, 209-224.

Gollo, L. L., Mirasso, C., and Villa, A. E. (2010). Dynamic control for synchronization of separated cortical areas through thalamic relay. Neuroimage doi: 10.1016/j.neuroimage.2009.11.058

Haider, B., and McCormick, D. A. (2009). Rapid neocortical dynamics: cellular and network mechanisms. Neuron 62 , 171-189.

Hendrickson, A. E., Wilson, J. R., and Ogren, M. P. (1978). The neuroanatomical organization of pathways between the dorsal lateral geniculate nucleus and visual cortex in old world and new world primates. J. Comp. Neurol. 182, 123-136.

Kramer, M.A., Roopun, A. K., Carracedo, L. M., Traub, R. D., Whittington, M. A., and Kopell, N. J. (2008). Rhythm generation through period concatenation in rat somatosensory cortex. PLoS Comput. Biol. 4, e1000169. doi:10.1371/journal.pcbi.1000169.

Otte, S., Hasenstaub, A., and Callaway, E. M. (2010). Cell type-specific control of neuronal responsiveness by gammaband oscillatory inhibition. J. Neurosci. 30, 2150-2159.

Reid, R. C., and Alonso, J.-M. (1995). Specificity of monosynaptic connections from thalamus to visual cortex. Nature 378, 281-284.

Reid, R. C., and Usrey, W. M. (2004). "Functional connectivity in the pathways from retina to striate cortex," in The Visual Neurosciences, Vol. 1, eds L. Chalupa and J. Werner (Cambridge, MA: MIT Press), 673-679.

Roopun, A. K., Kramer, M. A., Carracedo, L. M., Kaiser, M., Davies, C. H., Traub, R. D., Kopell, N. J., and Whittington, M. A. (2008). Period concatenation underlies interactions between gamma and beta rhythms in neocortex. Front. Cell. Neurosci. 2:1. doi:10.3389/neuro.03.001.2008. 
Sincich, L. C., and Horton, J. C. (2005). The circuitry of V1 and V2: integration of color, form, and motion. Annu. Rev. Neurosci. 28, 303-326.

Singer, W. (1993). Synchronization of cortical activity and its putative role in information processing and learning. Annu. Rev. Physiol. 55, 349-374.

Sohal, V. S., Zhang, F., Yizhar, O., and Deisseroth, K. (2009). Parvalbumin neurons and gamma rhythms enhance cortical circuit performance. Nature 459, 698-702.

Steriade, M. (2001). Impact of network activities on neuronal properties in corticothalamic systems. J. Neurophys. $86,1-39$.
Sun, W., and Dan, Y. (2009). Layer-specific network oscillation and spatiotemporal receptive field in the visual cortex. Proc. Nat. Acad. Sci. U.S.A. 106, 17986-17991.

Uhlhaas, P. J., Pipa, G., Lima, B., Melloni, L., Neuenschwander, S., Nikolic, D., and Singer, W. (2009). Neural synchrony in cortical networks: history, concept and current status. Front. Integr. Neurosci. 3:17. doi:10.3389/neuro.07.017.2009.

Vicente, R., Gollo, L. L., Mirasso, C. R., Fischer, I., and Pipa, G. (2008). Dynamical relaying can yield zero time lag neuronal synchrony despite long conduction delays.
Proc. Nat. Acad. Sci. U.S.A. 105, 17157-17162.

Womelsdorf, T., and Fries, P. (2007). The role of neuronal synchronization in selective attention. Curr. Opin. Neurobiol. 17, 1-7.

Yang, J.-W., Hanganu-Opatz, I. L., Sun, J.-J., and Luhmann, H. J. (2009). Three patterns of oscillatory activity differentially synchronize developing neocortical networks in vivo. J. Neurosci. 29, 9011-9025.

Conflict of Interest Statement: The authors declare that the research was conducted in the absence of any commercial or financial relationships that could be construed as a potential conflict of interest.

Received: 22 February 2010; paper pending published: 15March 2010; accepted: 16 March 2010; published online: 15 September 2010.

Citation: Briggs F and Usrey WM (2010)

Patterned activity within the local cortical architecture. Front. Neurosci. 4:18. doi: 10.3389/fnins.2010.00018

Copyright (c) 2010 Briggs and Usrey. This is an open-access article subject to an exclusive license agreement between the authors and the Frontiers Research Foundation, which permits unrestricted use, distribution, and reproduction in any medium, provided the original authors and source are credited. 